\title{
A Novel Preoperative Nomogram for Predicting Lymph Node Invasion in Renal Cell Carcinoma Patients Without Metastasis
}

This article was published in the following Dove Press journal: Cancer Management and Research

\author{
Pin $\mathrm{Li}^{1,2, *}$ \\ Cheng Peng ${ }^{3, *}$ \\ Yongpeng $\mathrm{Xie}^{4, *}$ \\ Lei Wang ${ }^{5}$ \\ Liangyou Gu' \\ Shengpan Wu' \\ Donglai Shen' \\ Yundong Xuan' \\ Xin $\mathrm{Ma}^{\mathrm{l}}$ \\ Xu Zhang' \\ 'Department of Urology, State Key \\ Laboratory of Kidney Diseases, The First \\ Medical Center of Chinese PLA General \\ Hospital, Beijing, People's Republic of \\ China; ${ }^{2}$ Department of Pediatric Urology, \\ Bayi Children's Hospital Affiliated to the \\ Seventh Medical Center of Chinese PLA \\ General Hospital, Beijing, People's \\ Republic of China; ${ }^{3}$ Department of \\ Urology, The Seventh Medical Center of \\ Chinese PLA General Hospital, Beijing, \\ People's Republic of China; ${ }^{4}$ Department \\ of Urology, The First Affiliated Hospital \\ of Chongqing Medical University, \\ Chongqing, People's Republic of China; \\ ${ }^{5}$ Chinese PLA 534 Hospital, Luoyang, \\ People's Republic of China \\ *These authors contributed equally to \\ this work
}

Correspondence: Xu Zhang; Xin Ma Department of Urology, Chinese PLA General Hospital, Beijing 100853, People's Republic of China

Tel +86- 10-6693-82I I; +86-10-6693-8008

Fax +86-10-6822-3567;

+86-10-6822-3575

Email xzhang@tjh.tjmu.edu.cn;

urologist@foxmail.com
Objective: To provide a preoperative predictive model to support clinical decision-making regarding the selection of in renal cell carcinoma (RCC) patients who will benefit the most from lymph node dissection.

Methods: This retrospective analysis enrolled $374 \mathrm{RCC}$ patients without distant metastasis who underwent surgical treatment from January 2006 to December 2017. The relationships between lymph node invasion (LNI) and age at surgery; gender; body mass index(BMI); the presence of clinical symptoms such as flank pain, hematuria or a palpable mass; clinical $\mathrm{T}$ stage (cT stage); clinical $\mathrm{N}$ stage (cN stage); and the results of routine hematological and serum biochemical analyses were investigated. All the variables were included in univariate and multivariate logistic regression analyses, and the significant variables were then included in a novel nomogram to predict the probability of LNI. Then, we calibrated the nomogram with an internal validation set.

Results: Six of eighteen variables were significant in the univariate logistic regression analysis. After multivariate logistic regression analysis, age at surgery ( $\mathrm{OR}=0.643,95 \% \mathrm{CI}: 0.421-0.975)$, cT stage $(\mathrm{OR}=3.034,95 \% \mathrm{CI}: 1.541-5.926)$, cN stage (OR=6.353, 95\% CI: 3.273-12.456), lymphocyte percentage $(\mathrm{OR}=0.481,95 \% \mathrm{CI}: 0.256-0.894)$, and the presence of clinical symptoms (OR=2.045, 95\% CI: 1.065-3.924) were independent predictors of LNI and were included in the nomogram. The $\mathrm{C}$-index of this nomogram was 0.824 .

Conclusion: Preoperative basic laboratory findings combined with the results of a physical examination and radiological examination can indicate the probability of LNI in RCC patients.

Keywords: kidney cancer, renal cell carcinoma, lymph node invasion, predictive nomogram

\section{Introduction}

Renal cell carcinoma (RCC) accounts for $2-3 \%$ of all systemic malignancies; that incidence is estimated to have increased to $3.7 \%$ in the United States in $2018 .{ }^{1}$ Approximately $30 \%$ of localized RCC patients will experience metastasis, of whom 2.7-10\% will have lymph node invasion (LNI), a widely acknowledged marker for poor prognosis in adults with RCC. ${ }^{2,3}$ Unlike bladder or prostate cancer, lymph node dissection (LND) in patients with RCC is still a subject of debate. The only level one evidence is the prospective randomized controlled EORTC 30881 study. It suggests that LND is not associated with oncologic outcomes in T1aN0M0 patients since the tumor-node-metastasis (TNM) classification system has modified thereafter. ${ }^{4}$ Previously, a series of retrospective studies and meta-analyses indicated 
that LND was unnecessary even in high-risk RCC patients. $^{5-7}$ However, several other retrospective studies showed that LND improved survival in RCC patients. ${ }^{8-10}$ Therefore, in the 2018 EAU guidelines for RCC, LND was recommended for high-risk RCC patients with an evidence level of $2 \mathrm{~b} .{ }^{11}$ Considering the strong oncological control benefit and provision of accurate information used for tumor classification, LND will continue to play an important role in locally advanced RCC surgery for a long time. As the positivity rate for LNI in RCC patients undergoing LND is only $4-8 \%$ among all non-metastatic RCC patients, there is an urgent need for a reliable predictive tool to estimate the risk of LNI in RCC patients to assist clinical decision-making with regard to selecting patients who are the most likely to benefit from LND.

\section{Data and Methods}

\section{Clinical Information}

In this study, 374 consecutive RCC patients who underwent radical nephrectomy with elective retroperitoneal LND or regional LND from January 2006 to December 2017 in our center were included. The clinical tumor staging was based on the 2018 American Joint Committee on Cancer (AJCC) staging system. The cT stage and $\mathrm{cN}$ stage were classified based on the results of magnetic resonance imaging (MRI) or computed tomography (CT) as assessed by two radiologists. $\mathrm{cN} 1$ indicates the presence of retroperitoneal or intraperitoneal lymph nodes larger than $1 \mathrm{~cm}$ on MRI/CT images. LNI (pN1) was confirmed in paraffin-embedded slices by two pathologists. Clinical symptoms were defined as flank pain, hematuria or a palpable mass. The laboratory examinations included routine hematological and serum biochemical analyses within one week before surgery. The tumor diameter was defined as the longest diameter through the tumor. Patients with incomplete information were excluded.

The study was undertaken with the approval of the PLAGH institutional ethics committee board. All patients signed the written consent forms.

\section{Analysis Methods}

Logistic regression analysis was employed to investigate the preoperative risk factors for LNI in RCC patients. The final pathological diagnosis of LNI was used as the dependent variable, and patients' clinical data before surgery were included as independent variables. These 18 variables were age at surgery, the presence of preoperative symptoms, gender, body mass index (BMI), Eastern
Cooperative Oncology Group performance status (ECOG PS), cT stage, cN stage, and hematological and serum biochemical analyses, namely, lymphocyte percent, monocyte percent, neutrophil count, hemoglobin level, serum creatinine, serum albumin level, free calcium level, platelet count, alkaline phosphatase level, lactate dehydrogenase level and the largest tumor diameter. Univariate logistic regression analysis was used to analyze the predictive value of different variables for LNI. Independent variables that demonstrated statistical significance on univariate analysis were taken into consideration in the multivariate logistic regression model. Then, the multivariate logistic regression analysis was conducted to identify significant variables for inclusion into the final model. In the univariate logistic regression analysis, we set age and the results of the hematological and serum biochemical analyses as continuous variables to maximize the data. For multivariate logistic regression analysis, we changed them from continuous variables to ordered categorical variables to simplify the model. We developed a nomogram with the final variables to visualize the predictive tool. The discrimination and calibration characteristics were internally validated. All statistical analyses were performed in the $\mathrm{R}$ software environment (version 3.4.0; http://r-project.org/), and $p<0.05$ was considered significant in all statistical analyses.

\section{Results \\ General Status}

Among the included patients, 63 (16.83\%) were classified as having LNI, and 311 patients were negative for LNI. Table 1 shows the distribution of clinical characteristics in the LNI and non-LNI groups.

\section{Statistical Analysis}

The univariate logistic regression analysis identified patient age at surgery, the presence of preoperative symptoms, largest tumor diameter, $\mathrm{cT}$ stage, $\mathrm{cN}$ stage, and serum biomarkers such as lymphocyte percent to be associated with LNI (Table 2). The multivariate logistic regression analysis indicated that age at surgery, cT stage, cN stage, lymphocyte percentage and the presence of preoperative symptoms were independent predictors for LNI. The ORs and 95\% CI were 0.643 (0.421-0.975), 3.034 (1.5415.926), 6.353 (3.273-12.456), 0.481 (0.256-0.894), and 2.049 (1.065-3.924), respectively. 
Table I Distribution of Independent Variables

\begin{tabular}{|c|c|c|c|}
\hline Parameter & All Cohort & LNI Group (63 Patients) & Non-LNI Group (3 I I Patients) \\
\hline Mean $( \pm S D)$ age at surgery & $53.56( \pm \mid 3.91)$ & $45.86( \pm 19.65)$ & $54.6 \mathrm{I}( \pm 12.27)$ \\
\hline \multicolumn{4}{|l|}{ Gender } \\
\hline Male & 183 & 24 & 159 \\
\hline Female & 95 & 12 & 73 \\
\hline Mean $( \pm S D)$ BMI $\left(\mathrm{kg} / \mathrm{m}^{2}\right)$ & $24.93( \pm 3.778)$ & $24.79( \pm 3.759)$ & $24.96( \pm 3.790)$ \\
\hline Preoperative symptoms & $70(25.18 \%)$ & $20(55.56 \%)$ & $50(21.55 \%)$ \\
\hline Mean $( \pm S D)$ largest tumor diameter $(\mathrm{cm})$ & $5.038( \pm 3.03)$ & $6.757( \pm 3.721)$ & $4.819( \pm 2.837)$ \\
\hline Mean $( \pm S D)$ lymphocyte count & $0.2805( \pm 0.09373)$ & $0.2323( \pm 0.09502)$ & $0.2875( \pm 0.09324)$ \\
\hline Mean $( \pm S D)$ neutrophil count & $1.6256( \pm 0.1017)$ & $0.6104( \pm 0.1015)$ & $1.782( \pm 0.1009)$ \\
\hline Mean $( \pm S D)$ monocyte count & $0.06637( \pm 0.02008)$ & $0.06477( \pm 0.02146)$ & $0.06650( \pm 0.01983)$ \\
\hline Mean $( \pm S D)$ serum albumin & $39.0385( \pm 11.17)$ & $34.02( \pm 11.04)$ & $39.72( \pm 11.99)$ \\
\hline Mean $( \pm S D)$ free calcium & $2.868( \pm 0.06162)$ & $1.897( \pm 0.06214)$ & $3.024( \pm 0.06162)$ \\
\hline Mean $( \pm S D)$ hemoglobin & $132.3( \pm 22.82)$ & $121.2( \pm 23.59)$ & $133.7( \pm 23.80)$ \\
\hline Mean $( \pm \mathrm{SD})$ platelet & $233.2( \pm 75.52)$ & $240.3( \pm 75.42)$ & $239.8( \pm 76.33)$ \\
\hline Mean $( \pm S D)$ serum creatinine & $77.03( \pm 23.56)$ & $77.31( \pm 25.06)$ & $76.98( \pm 23.30)$ \\
\hline Mean $( \pm S D)$ alkaline phosphatase & $62.13( \pm 36.07)$ & $62.00( \pm 38.5 I)$ & $75.15( \pm 30.38)$ \\
\hline Mean $( \pm S D)$ lactate dehydrogenase & $192.5( \pm 53.75)$ & $|7| .0( \pm 6 \mid .78)$ & $155.5( \pm 5 \mid .60)$ \\
\hline \multicolumn{4}{|l|}{ cT stage } \\
\hline $\mathrm{I}-2$ & 235 & 19 & 216 \\
\hline $3-4$ & 43 & 17 & 26 \\
\hline cN stage & 64 & 33 & 31 \\
\hline \multicolumn{4}{|l|}{ ECOG PS } \\
\hline I & 257 & 34 & 223 \\
\hline$>1$ & 21 & 2 & 19 \\
\hline
\end{tabular}

Note: Age at surgery, gender, BMI, presence of clinical symptoms, the results of routine hematological, serum biochemical analyses and radiological examinations were included as independent variables.

Univariate and multivariate logistic regression analyses were performed and the OR and $p$-value was calculated. We selected the $95 \%$ confidence interval.

Based on all the clinical characteristics available before surgery, a novel predictive tool was developed to predict LNI (Figure 1). The C-index of this nomogram was 0.824 . The nomogram-predicted probability was explored graphically using a calibration plot with the bootstrap method (Figure 2).

\section{Nomogram Instructions}

To obtain the nomogram-predicted probability of nodal metastasis after surgery, locate the patient parameters on each axis. Draw a vertical line to the "Points" for all variables. Locate the sum on the "Total Points" line to assess the individual on the LNI Probability line.

The calibration plot was calculated with the bootstrap methods (1000 times).

\section{Discussion}

Several prediction tools have been proposed to predict the patients who will benefit the most from LND worldwide. Blute et al evaluated 1652 kidney cancer patients who underwent radical nephrectomy and identified nuclear grade 3 or 4 , the presence of a sarcomatoid component, a tumor size $>10 \mathrm{~cm}$, tumor stage pT3-pT4, and histological tumor necrosis as risk factors 
Table 2 Univariate and Multivariate Logistic Regression Analysis

\begin{tabular}{|c|c|c|c|c|}
\hline \multirow[t]{2}{*}{ Parameter } & \multicolumn{2}{|l|}{ Univariate Analysis } & \multicolumn{2}{|c|}{ Multivariate Analysis } \\
\hline & OR(95\% Cl) & p-value & OR(95\% Cl) & p-value \\
\hline Age at surgery & $0.974(0.956-0.993)$ & 0.00632 & $0.643(0.42 I-0.975)$ & 0.0387 \\
\hline Gender & $0.912(0.523-1.622)$ & 0.749 & & \\
\hline BMI $\left(\mathrm{kg} / \mathrm{m}^{2}\right)$ & $0.988(0.907-1.072)$ & 0.775 & & \\
\hline Symptoms & $2.964(1.804-5.540)$ & 0.000142 & $2.049(1.065-3.924)$ & 0.0305 \\
\hline Largest diameter $(\mathrm{cm})$ & I.I28(I.036-I.229) & 0.00517 & & \\
\hline Lymphocyte percent & $0.367(0.216-0.613)$ & 0.000155 & $0.48 I(0.256-0.894)$ & 0.0217 \\
\hline Monocyte percent & $5.344(4.77 \mathrm{E}-06-3.26 \mathrm{E}+06)$ & 0.809 & & \\
\hline Neutrophil count & $0.988(0.929-1.013)$ & 0.787 & & \\
\hline Serum albumin & $0.991(0.954-1.017)$ & 0.585 & & \\
\hline Free calcium & $0.998(0.966-1.011)$ & 0.796 & & \\
\hline Hemoglobin & $0.99 \mid(0.98 I-1.003)$ & 0.122 & & \\
\hline Serum creatinine & $0.915(0.988-1.012) 1.0120$ & 0.142 & & \\
\hline Platelet & $1.000(0.997-1.004)$ & $0.85 I$ & & \\
\hline Alkaline phosphatase & $1.982(0.908-4.455)$ & 0.0914 & & \\
\hline Lactate dehydrogenase & $0.957(0.460-2.038)$ & 0.908 & & \\
\hline cT stage & $4.854(2.685-8.780)$ & I.57E-07 & $3.034(1.54 I-5.926)$ & 0.00118 \\
\hline cN stage & $9.934(5.387-18.613)$ & $3.32 \mathrm{E}-13$ & $6.353(3.273-12.456)$ & $5.33 \mathrm{E}-08$ \\
\hline ECOG PS & I.827(0.772-3.988) & 0.146 & & \\
\hline
\end{tabular}

for LNI in multivariate analysis. The symptoms of complications, distant metastasis, and positive tumor thrombus in the inferior vena cava are also risk factors for LNI. ${ }^{12}$ However, these pathological indicators can only be assessed after surgery, which severely limited their application. Capitanio et al provided a presurgery model to predict the need for LND. They concluded that the tumor stage, clinical nodal status, metastases at diagnosis, and clinical tumor size are informative independent predictors. Although they provided a model for surgery decision making, most metastatic RCC patients will not even undergo an operation. ${ }^{13}$ Then, Babaian et al provided a novel predictive model in $2015 .^{2}$ Their model includes local symptoms, ECOG-PS, cN stage, and the lactate dehydrogenase level as risk factors. Although their model was found to have excellent prediction efficiency, it appears to not work well in our patients. We were disappointed that the lactate dehydrogenase level, as well as the classic prognostic factors alkaline phosphatase, free calcium, serum creatinine, and hemoglobin levels, were not different between the two groups in our cohort. These findings limit the application of Babaian's predictive model in our center.

In our cohort, we developed a model based entirely on preoperative routine hematological and serum biochemical analyses, radiological examinations and physical examinations. Relatively younger RCC patients have a higher incidence of LNI than relatively older patients; this finding is common in RCC, as Sánchez-Ortiz et al noted. ${ }^{14}$ This could be attributed to the highly malignant biological behavior (sarcoma and necrosis) of RCC in relatively younger patients. Lymphocyte percentage is a traditional biomarker for prognosis in RCC patients, and we found that a high lymphocyte percentage may be associated with a lower probability of LNI. This phenomenon is probably the result of the tumor-associated systemic inflammatory reactions because platelet-tolymphocyte ratios (PLRs), neutrophil-to-lymphocyte ratios (NLRs), and lymphocyte-to-monocyte ratios (LMRs), have also been reported as biomarkers in many tumors. ${ }^{15,16}$ The $\mathrm{cN}$ stage showed the strongest predictive value, with an OR of 6.353 in our results, which was consistent with Babaian's result; ${ }^{2}$ however, the OR for cT stage was 3.034, which was not found in their study.

This study also has limitations. First, the heterogeneity of surgery techniques among the patients we enrolled may have influenced the LND technical standard. The patients admitted from 2006 to 2017 underwent different approaches to nephrectomy, such as open surgery, laparoscopic surgery, and robot-assisted surgery. In addition, laparoscopic and robot-assisted surgery can be further divided into transabdominal preperitoneal or 
Points

$\begin{array}{llllllllll}0 & 10 & 20 & 30 & 40 & 50 & 60 & 70 & 80 & 90\end{array}$

Age at surgery

$>60 \quad 40-60<20-40$

Lymphocyte percent

Normal

High Low

cT stage

$$
\text { T1-T2 }
$$

T3-T4

cN stage

0

Symptoms

absent presence

Total Points

\begin{tabular}{llllllll}
\hline 1 & 100 & 150 & 200 & 250 & 300 & 350 & 400
\end{tabular}

LNI Probability

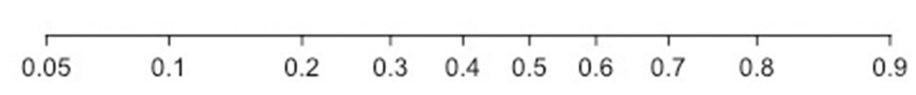

Figure I Nomogram depicting the probability of LNI.

retroperitoneal approaches. Capitanio et al proposed that the number of lymph nodes was associated with cancerspecific survival (CSS) or metastatic progression-free survival (MPFS), so different LND techniques may lead to different clinical outcomes. ${ }^{17}$ Moreover, the involvement of interaortocaval LNI without regional hilar LNI is reported in up to $35-45 \%$ of cases, ${ }^{18}$ which further complicates the LND strategy. Second, the scope of LND also partially depends on the surgeon's preference and intraoperative decisions. Thus, we did not distinguish between extended LND and LND. Third, we did not perform a radiological re-review of the preoperative imaging. Fourth, as all participants in our research were under
LND, this group may have not enough representation for the whole RCC patients. Meanwhile, as we do not have a standard LND panel before surgery, some of the LND was passively performed (for example the LN stand before the necessary artery), this also added the selection bias of this research. Finally, this study had a retrospective singlecenter design. Although we enrolled 374 patients, the sample size was too small. We hope that this model will be externally validated by other centers worldwide.

\section{Conclusion}

Age at surgery, lymphocyte percentage, cT stage, cN stage, and the presence of preoperative symptoms were 


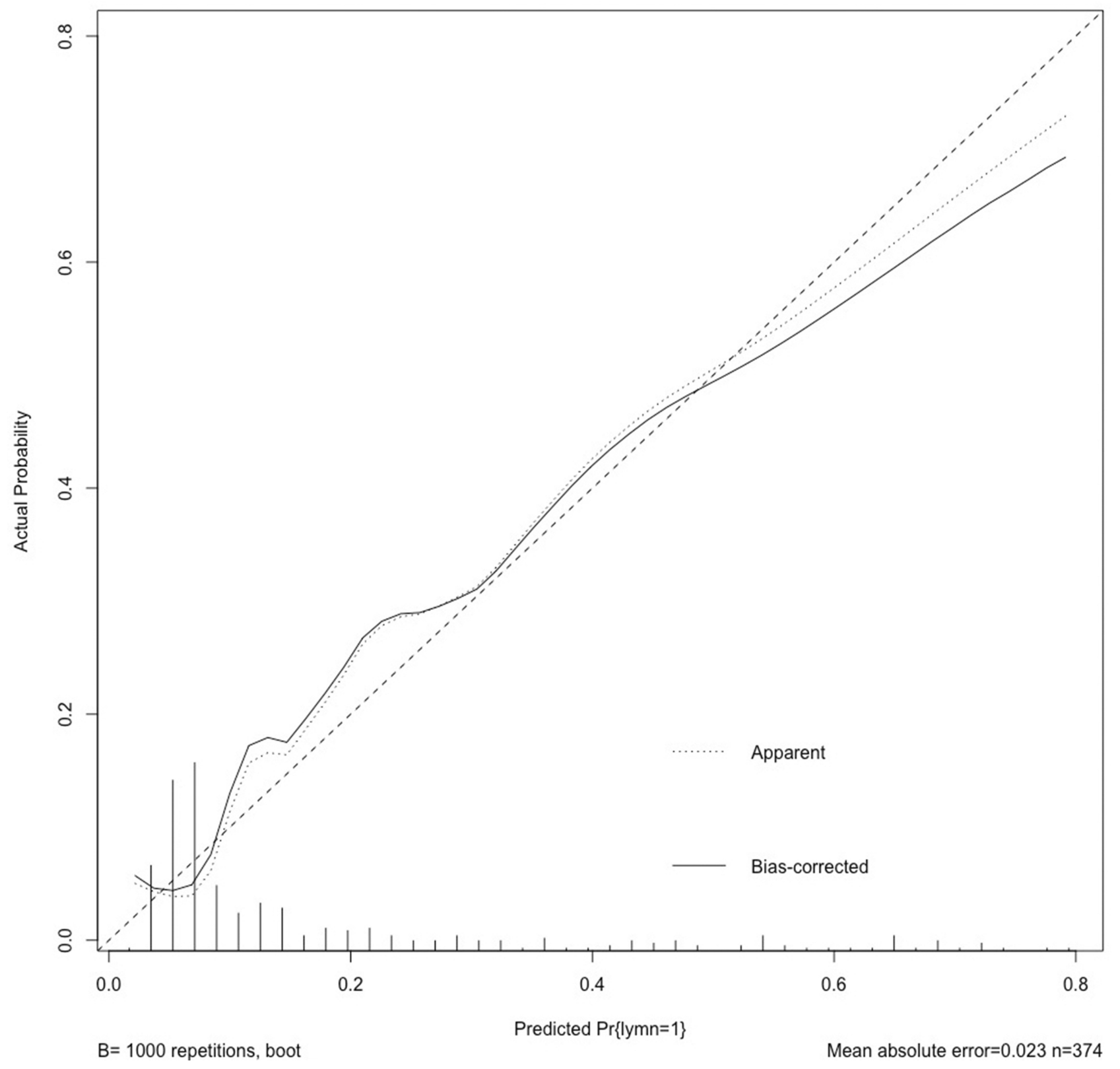

Figure 2 Calibration plot depicting the relationship between the predicted and actual probabilities.

independent predictors of LNI in our cohort of RCC patients. Preoperative parameters can indicate the probability of LNI in patients with RCC. We suggest that LND be considered as a treatment strategy in patients with a high probability of LNI.

\section{Ethical Approval}

All procedures performed in studies involving human participants were in accordance with the ethical standards of the institutional and the ethical committee of Chinese PLA General Hospital with the 1964 Helsinki declaration and its later amendments or comparable ethical standards.

\section{Informed Consent}

Informed consent was obtained from all individual participants included in the study.

\section{Acknowledgments}

This work was supported by the People's Republic of China and the National High Technology Research and Development Program ("863" Program) of China: the 
screening and clinical validation of characteristic protein biomarkers in renal cancer based on a large-scale biobank (2014AA020607) and the National Natural Science Foundation of China(Grant No. 81702492).

\section{Author Contributions}

All authors contributed to data analysis, drafting and revising the article, gave final approval of the version to be published, and agree to be accountable for all aspects of the work.

\section{Disclosure}

The authors report that they have no conflicts of interest in this work.

\section{References}

1. Siegel RL, Miller KD, Jemal A. Cancer statistics, 2018. CA Cancer J Clin. 2018;68:24. doi:10.3322/caac.21442

2. Babaian KN, Kim DY, Kenney PA, et al. Preoperative predictors of pathological lymph node metastasis in patients with renal cell carcinoma undergoing retroperitoneal lymph node dissection. $J$ Urol. 2015;193:1101-1107. doi:10.1016/j.juro.2014.10.096

3. Hsieh JJ, Purdue MP, Signoretti S, et al. Renal cell carcinoma. Nat Rev Dis Primer. 2017;3:17009. doi:10.1038/nrdp.2017.9

4. Capitanio U, Leibovich BC. The rationale and the role of lymph node dissection in renal cell carcinoma. World J Urol. 2017;35:497-506. doi:10.1007/s00345-016-1886-3

5. Feuerstein MA, Kent M, Bazzi WM, Bernstein M, Russo P. Analysis of lymph node dissection in patients with $\geq 7-\mathrm{cm}$ renal tumors. World J Urol. 2014;32:1531-1536. doi:10.1007/s00345-013-1233-x

6. Gershman B, Thompson RH, Moreira DM, et al. Radical nephrectomy with or without lymph node dissection for nonmetastatic renal cell carcinoma: a propensity score-based analysis. Eur Urol. 2017;71:560-567. doi:10.1016/j.eururo.2016.09.019

7. Bekema HJ, MacLennan S, Imamura M, et al. Systematic review of adrenalectomy and lymph node dissection in locally advanced renal cell carcinoma. Eur Urol. 2013;64:799-810. doi:10.1016/j.eururo. 2013.04.033
8. Whitson JM, Harris CR, Reese AC, Meng MV. Lymphadenectomy improves survival of patients with renal cell carcinoma and nodal metastases. J Urol. 2011;185:1615-1620. doi:10.1016/j.juro.2010. 12.053

9. Pantuck AJ, Zisman A, Dorey F, et al. Renal cell carcinoma with retroperitoneal lymph nodes: role of lymph node dissection. $J$ Urol. 2003;169:2076-2083. doi:10.1097/01.ju.0000066130.27119.1c

10. Crispen PL, Breau RH, Allmer C, et al. Lymph node dissection at the time of radical nephrectomy for high-risk clear cell renal cell carcinoma: indications and recommendations for surgical templates. Eur Urol. 2011;59:18-23. doi:10.1016/j.eururo.2010.08.042

11. Ljungberg B, Albiges L, Bensalah K, et al. EAU guildelines on renal cell carcinoma 2018. n.d.

12. Blute ML, Leibovich BC, Cheville JC, Lohse CM, Zincke H. A protocol for performing extended lymph node dissection using primary tumor pathological features for patients treated with radical nephrectomy for clear cell renal cell carcinoma. $J$ Urol. 2004;172:465-469. doi:10.1097/01.ju.0000129815.91927.85

13. Capitanio U, Abdollah F, Matloob R, et al. When to perform lymph node dissection in patients with renal cell carcinoma: a novel approach to the preoperative assessment of risk of lymph node invasion at surgery and of lymph node progression during follow-up: how to predict the risk of LNI or LN progression in RCC. BJU Int. 2013;112:E59-E66. doi:10.1111/bju.12125

14. Sánchez-Ortiz RF, Rosser CJ, Madsen LT, Swanson DA, Wood CG. Young age is an independent prognostic factor for survival of sporadic renal cell carcinoma. J Urol. 2004;171:2160-2165. doi:10.1097/ 01.ju.0000125487.96469.2e

15. Tan D, Fu Y, Tong W, Li F. Prognostic significance of lymphocyte to monocyte ratio in colorectal cancer: a meta-analysis. Int J Surg. 2018;55:128-138. doi:10.1016/j.ijsu.2018.05.030

16. Ming L, Jiang Z, Ma J, Wang Q, Wu F, Ping J. Platelet-tolymphocyte ratio, neutrophil-to-lymphocyte ratio, and platelet indices in patients with acute deep vein thrombosis. Vasa. 2018;47(2): 143-147.

17. Capitanio U, Suardi N, Matloob R, et al. Extent of lymph node dissection at nephrectomy affects cancer-specific survival and metastatic progression in specific sub-categories of patients with renal cell carcinoma (RCC): effect of LND extent on RCC-specific survival and metastatic progression. BJU Int. 2014;114:210-215. doi:10.1111/ bju. 12508

18. Capitanio U, Becker F, Blute ML, et al. Lymph node dissection in renal cell carcinoma. Eur Urol. 2011;60:1212-1220. doi:10.1016/j. eururo.2011.09.003

\section{Publish your work in this journal}

Cancer Management and Research is an international, peer-reviewed open access journal focusing on cancer research and the optimal use of preventative and integrated treatment interventions to achieve improved outcomes, enhanced survival and quality of life for the cancer patient.
The manuscript management system is completely online and includes a very quick and fair peer-review system, which is all easy to use. Visit http://www.dovepress.com/testimonials.php to read real quotes from published authors. 